\title{
CANOMAD syndrome
}

INSERM

\section{Source}

INSERM. (1999). Orphanet: an online rare disease and orphan drug data base. CANOMAD syndrome. ORPHA:71279

A rare chronic immune-mediated polyneuropathy characterized by a progressive disabling neuropathy with marked gait disturbance primarily due to sensory ataxia with concurrent cranial neuropathies (internal or external ophthalmoplegia, dysphagia, dysarthria, or facial weakness) and anti-disialosyl IgM antibodies. 